INTERNATIONAL DESIGN CONFERENCE - DESIGN 2018

https://doi.org/10.21278/idc.2018.0449

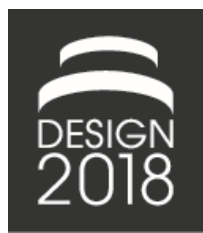

\title{
A METHODICAL APPROACH FOR THE TECHNOLOGICAL ASSESSMENT OF JOINING TECHNOLOGIES - OPTIMIZED DECISION- MAKING IN CAR BODY DEVELOPMENT
}

\author{
S. A. Choudry, S. Haass, U. Alber and D. Landgrebe
}

\begin{abstract}
Lightweight solution lead to a diversity of joining technologies in car body development. To date, the decision-making for joining technologies is based on the expertise of the developer; a standardized selection procedure does not exist. The focus of the extant literature mostly lies on economic criteria. Thus, an assessment of the technological potential of the technologies cannot be made. This research presents an approach for the technological assessment. By considering besides economic also technological criteria, the approach allows an optimized selection-process of joining technologies.
\end{abstract}

Keywords: technology development, design support system, car body development, joining technologies, optimisation

\section{Introduction}

Car body development as one of the most important steps in the automobile value chain is facing considerable challenges such as growing competition, stricter environmental regulations, as well as rising production costs. To manufacture innovative car body and lightweight structures various materials and configurations are being used (Teske et al., 2013).

However, the specific characteristics of individual materials are presenting new challenges, particularly in the joining technology where technologies face numerous restrictions. Thus, there is a need to develop new technologies for different materials (e.g., for joining aluminum and carbon fiber) which on the other hand leads to a growing diversity of joining technologies (Hahn et al., 2013). The diversity necessitates analyzing the economic, technological, and ecological potential of a technology for component-specific applications.

To date, the decision-making of the optimum joining technology in the automotive sector is mostly based on the expertise of the developer; a standardized selection procedure considering economic, ecological, and technological criteria does not exist. The extant literature focuses mostly on economic criteria. Due to the non-transparency of the overall expenditure and the benefit of joining technologies for individual applications, no statement can be made about the technological or ecological impact of a joining technology (Choudry et al., 2018). Particularly the technological criteria, such as strength or flange width, are essential to ensure the technological feasibility and a certain standard of quality. Hence, an essential part of this research is the specification of a methodical approach for the assessment of technological criteria to complement the multi-criteria approach, which considers besides economic and ecological also technological criteria.

For the technological assessment, a weighting and evaluation model has been developed. The evaluation model evaluates joining technologies. The weighting model, on the other hand, indicates the importance 
of each criterion. The findings of the developed models are consolidatet in performance indicators, which represent the technological value of a joining technology. By considering besides the existing economic criteria also the technological criteria, the approach allows an overall transparent and optimized selection-process of joining technologies to identify the optimum solution already in the early stage of car body development.

\section{Literature review}

Within a previous study, relevant research models for the assessment of manufacturing technologies were analyzed to identify the requirements for a multidimensional assessment of joining technologies (Choudry et al., 2018). The consideration of monetary and non-monetary factors represented essential requirements. Basically, a transformation of non-monetary in monetary factors would be possible, but the aggregation of the factors would lead to additional fuzziness (Breiing and Knosala, 1997).

Hence, non-monetary factors are considered independently. While the economic criteria include all monetary factors, non-monetary factors can be technological or ecological. Since the emphasis of this research is the technological assessment, further analysis of non-monetary factors is constrained to technological criteria. However, the technological assessment requires two basis-models which will be analyzed: the evaluation model to characterize joining technologies and the weighting model to consider the realistic impact of criteria with individual weighting factors.

\subsection{Evaluation model for technological criteria}

Within the framework of a benchmark study, relevant evaluation models for technological criteria were analyzed and verified with derived requirements conducted by a stakeholder analysis. Central requirements were the transferability, the consideration of non-monetary quantitative, as well as, qualitative factors, weighting, and differentiation of criteria.

Table 1. Excerpt of relevant evaluation models

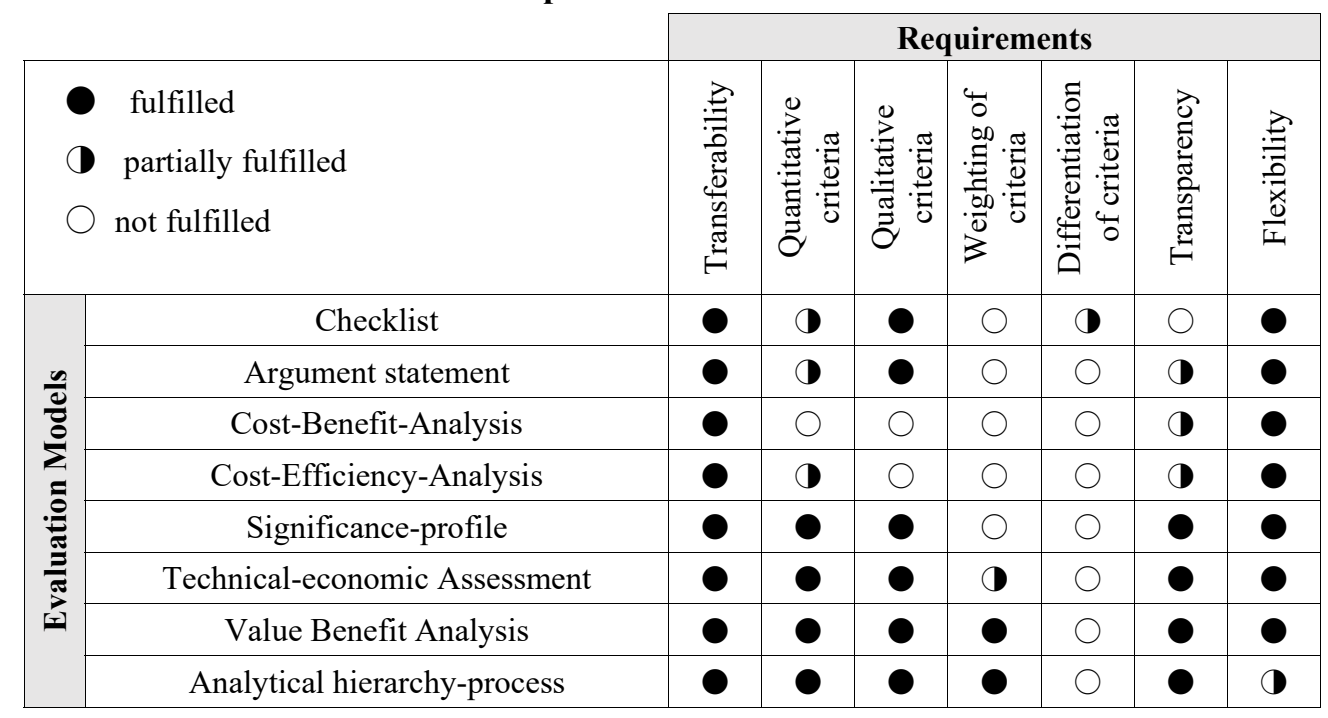

Transferability requires a universal validity of the approach due to the individual characteristics of technologies. To indicate a genuine value of non-monetary criteria, a consideration of qualitative and quantitative factors is necessary. Qualitative factors, which are not measurable, take lingual criteria such as "high complexity" into account, requiring a high evaluation effort due to their subjectivity.

Quantitative factors, on the other hand, are objective and measurable such as "flange width," hence, associated with less effort (Breiing and Knosala, 1997). Both, qualitative and quantitative factors have individual importance necessitating a weighting model to indicate the technological relevance of each criterion. The differentiation of global and local criteria, as well as binding constraints, is essential for an individual evaluation of the components. Global criteria (e.g., usability) are component-independent 
whereas local criteria can vary for each component (e.g., strength). Binding constraints (e.g., joining Aluminum with Steel) support a more efficient selection process since neither the technological nor the economic value is relevant if the joining technology does not allow joining of composite materials. Further requirements are the transparency and the flexibility to expand the evaluation in future. For a benchmark, relevant evaluation models are compared with the conducted requirements to identify the potential of the existing models (Table 1).

The benchmark shows that only the Technical-economic assessment, the value-benefit-analysis, and the analytical hierarchy process can nearly satisfy all requirements. The technical-economic assessment considers monetary as well as non-monetary criteria individually for the calculation of two performance indicators: one for the technological value and the other for costs (VDI 2225, 1998). The value-benefitanalysis, on the other hand, is based on the multi-attributive utilization theory where the collective goal consists of several individual sub-goals which are analyzed and weighted separately. The outcome of the analysis is the aggregation of all weighted sub-goals; hence, the method is only applicable to identify a preference order (Kühnapfel, 2014).

The analytical hierarchy process is a mathematical method for organizing and analyzing complex decision problems. For the evaluation, the problem is split and arranged into a hierarchy of subproblems, which can be analyzed independently. The comparison of the several sub-problems is based on the pairwise comparison, where a numerical weight can be derived for each element in the hierarchy to calculate and identify the priorities for the decision alternatives (Saaty, 2008).

However, none of the reviewed evaluation models can completely meet all requirements. Particularly the differentiation of criteria according to their relevance and the weighting of non-monetary criteria has rarely been analyzed for the assessment of joining technologies. Hence, a new approach for the evaluation of joining technologies is required.

\subsection{Weighting model for technological criteria}

Similar to the benchmark of evaluation models for technological criteria, relevant weighting models were analyzed and verified with the requirements conducted by a stakeholder analysis to identify the potential of existing models. A transparent, clear, realistic, and objective weighting of criteria, as well as, low time-effort and complexity of the weighting model were identified as central requirements. The benchmark shows that a few existing models are able to satisfy the requirements partially (Table 2).

Table 2. Excerpt of relevant weighting models

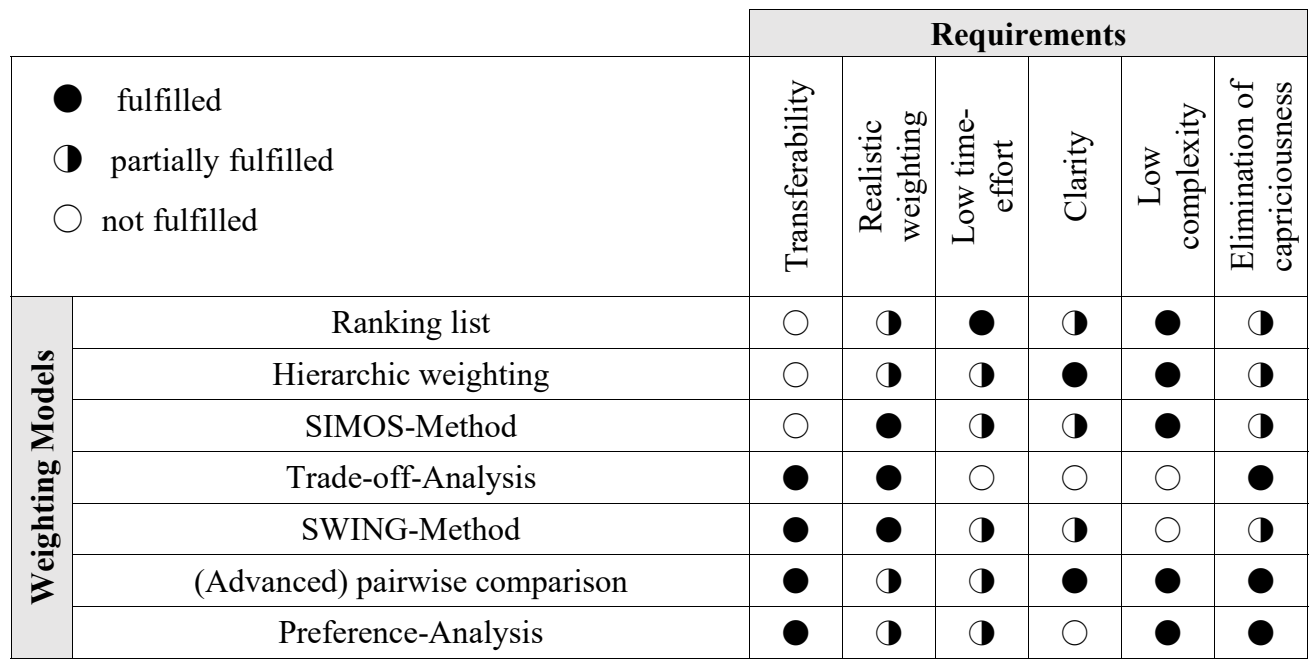

The SIMOS-method, for instance, allows a weighting of criteria by arranging the order of cards to demonstrate the preferences (Siskos and Tsotsolas, 2015). Blank cards between criteria can be used to highlight the distance of preference to the next criteria. With the total number of cards and the ranking 
order, the weighting for the criteria can be calculated. A weakness of the method is the transparency and also the complexity for a higher number of criteria.

Other recognized weighting methods are the pairwise comparison and preference-analysis where each criterion is compared to the others to identify the preferences. While the pairwise comparison allows equal weighting, the preference-analysis instructs a decision between " 0 " for less important and " 1 " for more important solely. The calculation of the weighting is based on the rank order (Breiing and Knosala, 1997). A weakness of both methods is the missing possibility to highlight the distance of preference between two criteria. Hence, only an ordinal ranking can be identified. For the pairwise comparison, which uses a matrix for more clearness, also exists an advanced version of Saaty which uses a scale of nine steps to allow a more precise differentiation of the comparison (Fink et al., 2006). However, similar to the comparison in section A none of the reviewed weighting models can completely meet all requirements. Hence, a new approach for the weighting of technological criteria is required.

\section{Development of the evaluation and weighting method}

Since the requirements identified in section two for the technological assessment of joining technologies in car body development are very specific, extant evaluation and weighting models are not able to meet the completely. Hence, a new approach is presented.

\subsection{Evaluation model for technological criteria}

The structure of the evaluation is designed in four steps: Identification and characterization of criteria, defining a standardized scaling, evaluation of the criteria, and finally the transformation of the characterized criteria into the standardized scaling (Figure 1).

1. Identification \& characterization

\section{Standardized} scaling
3. Evaluation
4. Transformation

Figure 1. Structure of the evaluation model

The first step involves the identification and characterization of relevant criteria. The identification of relevant criteria can be realized by a product analysis or interviews; however, a characterization of criteria is more complicated. Initially, a differentiation between quantitative and qualitative criteria has to be made. For example, flange width is expressed by a numeric value and represents a quantitative criterion. Process complexity, on the other hand, is described by lingual values such as "low" or "high"; thus, the criterion is a qualitative factor (Table 3). An additional differentiation for the characterization of criteria is required for global and local criteria, as well as binding constraints. Global criteria are due to their independence to the product constantly static whereas local criteria and binding constraints can be derived from the product specification and therefore may show a dynamic behavior.

Table 3. Exemplary characterization of criteria

\begin{tabular}{|c|c|c|}
\hline Criteria & Nature & Cluster \\
\hline Flange width & Quantitative & Local criteria \\
\hline Complexity & Qualitative & Global criteria \\
\hline Accessibility & Quantitative & Binding constraint \\
\hline
\end{tabular}

The second step defines a standardized scaling system with a span from zero to nine for the criteria. For the evaluation exist four different scaling classes: nominal, ordinal, interval, and ratio scale (Nedjah and Macedo Mourelle, 2005). The nominal scale, for example, can be used for qualitative criteria which have two or three possible options to describe the situation through lingual terms (e.g., "yes" or "no"). The interval scale can be used for qualitative criteria, which have more than three possible options, where the scaling points have an equal distance to each other. An adequate scaling span, in this case, was required to allow an accurate differentiation of the evaluation (Miller, 1994). Since the middle of a scale is often used as an escape way, an even number of scaling points has been chosen to avoid neutral 
evaluation (Porst, 2014). The deployed Likert-Scaling, which uses for both starting and endpoint linguistic terms (e.g., "very low complexity" and "very high complexity") complements the interval scale (Tullis and Albert, 2008). Quantitative criteria, in turn, are expressed on a ratio scale with a metric scale allowing an interpretation of the differences between each point and the ratio.

The third step is the technological evaluation, where a joining technology has to be characterized with a catalog of questions and a standardized scale. An excerpt of representative questions and scale alternatives for the technological evaluation is shown below (Figure 2).

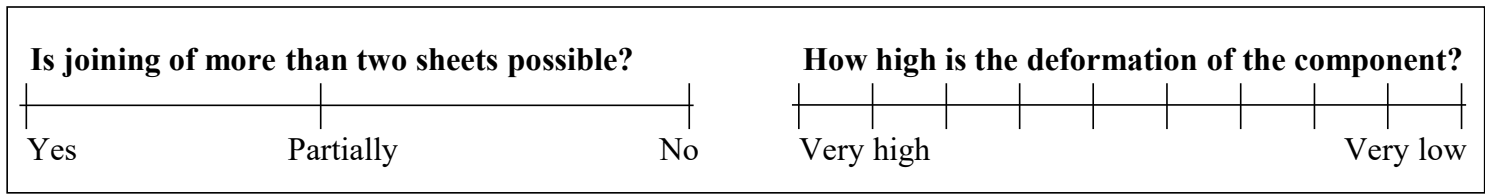

Figure 2. Technological evaluation: Excerpt of exemplary questions

The last step is the transformation of the given answers from step three to normalized scaling points to ensure a comparableness of the criteria. The transformation function varies for qualitative and quantitative criteria; however, the minimum scaling value $\left(S P_{\min }\right)$ for the functions is always zero and the optimum scaling value $\left(S P_{\max }\right)$ nine. For quantitative criteria exist a linear decreasing preferencefunction (a) and a linear increasing preference-function (b) (Figure 3).

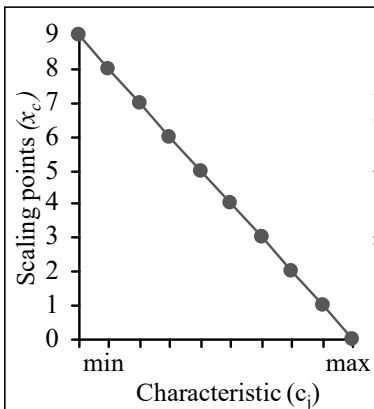

(a)

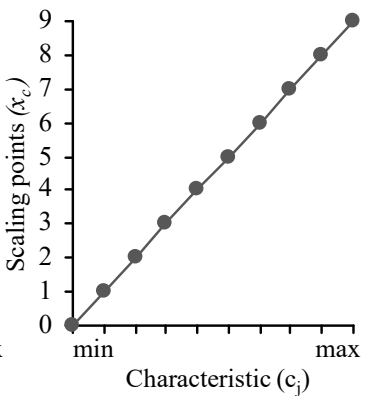

(b)

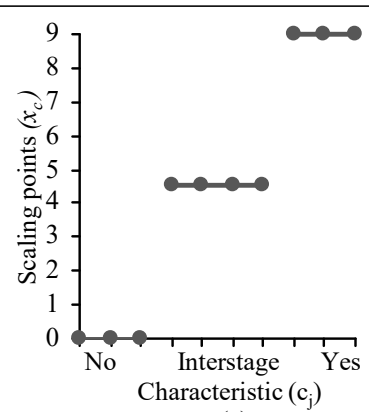

(c)

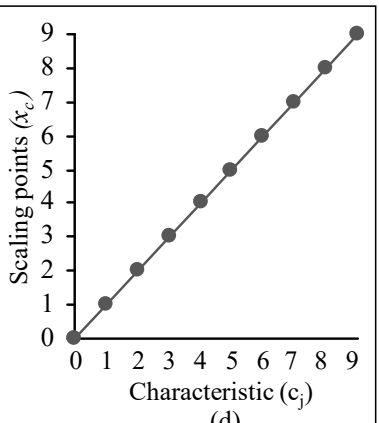

(d)

Figure 3. Overview of the preference functions with (a) decreasing function; (b) increasing function; (c) step-function; (d) linear preference function

While the decreasing preference-function is required for criteria which have to be minimized (e.g., flange width), the increasing preference-function is used for criteria which have to be maximized (e.g., strength). The calculation of the transformation function for the slope $s_{c}$ is described in (1), whereby the minimum $v_{\min }$ and the maximum characteristic $v_{\max }$ represent the poorest and the optimum value, which a joining technology can receive.

$$
S_{c}=\frac{v_{\min }-v_{\max }}{S P_{\min }-S P_{\max }}
$$

Finally, with (2) the evaluated characteristic of the joining technology $v_{T}$ can be transformed to the normalized scaling point $x_{c}$ where $c$ is the set of criteria, and $T$ stands for the index of alternative technologies. While the first formula is valid for criteria with a decreasing function, the second formula calculates the scaling points for an increasing function.

$$
x_{c, T}=\frac{V_{c, T}-S P_{\max }}{s_{c}}(a) \quad \vee \quad x_{c, T}=\frac{V_{c, T}-S P_{\min }}{s_{c}}(b)
$$

For the transformation of qualitative criteria, on the other hand, are also two functions available (Figure 3). Criteria with a maximum of three possible characteristics can be described with a step-function (c) where each characteristic is correlated to a numeric value. The step-function is exemplarily described as zero, four, and nine to achieve a negative tendency for the middle characteristic. For qualitative criteria 
with more than three possible characteristics exist a linear preference-function (d). Since the normalized scaling points are already determined during the evaluation, an additional transformation is not required.

\subsection{Weighting method for technological criteria}

The weighting model is in contrast to the evaluation model technology-independent. The weighting model uses the pairwise comparison, which has been introduced in section two, to identify and quantify the importance of each criterion. The matrix excerpt lists all criteria vertically and horizontally to compare criteria $i$ with criteria $j$ (Table 4).

Table 4. Excerpt of pairwise comparison

\begin{tabular}{|c|c|c|c|c|}
\hline $\boldsymbol{i}$ & $\mathbf{A}$ & $\mathbf{B}$ & $\mathbf{C}$ & Frequency (f) \\
\hline $\mathrm{A}$ & - & $a_{12}$ & $a_{13}$ & $\sum_{j=1}^{n} a_{1 j}$ \\
\hline $\mathrm{B}$ & $a_{21}$ & - & $a_{23}$ & $\sum_{j=1}^{n} a_{2 j}$ \\
\hline $\mathrm{C}$ & $a_{31}$ & $a_{32}$ & - & $\sum_{j=1}^{n} a_{3 j}$ \\
\hline
\end{tabular}

If criteria $i$ has more importance than criteria $j$ the field $a_{i j}$ receives the value one, with less importance the value zero, and with equal importance the value 0.5. Conversely, the values below the diagonal for field $a_{j i}$ are calculated with (3). Since the sum of $a_{i j}$ and $a_{j i}$ for the compared criteria is always one, values below the diagonal do not need further attention (Breiing and Knosala, 1997). In case, criterion $i$ and $j$ are identical (diagonal), the comparison indicates a null set.

$$
a_{j i}=1-a_{i j} \forall i \neq j \quad \wedge \quad a_{j i}=\{\} \forall i=j
$$

A consistency-check has been integrated to verify the transitivity, minimizing errors and effort for the comparison. A binary relation $R$ over a set $M$ is transitive if the criterion $a$ is related to $b$, and $b$, in turn, is related to $c$ which indicates according to (4) a relation between criterion $a$ and $c$ (Fodor and Roubens, 2011).

$$
\forall a, b, c \in M: a R b \wedge b R c \Rightarrow a R b
$$

After adding up the frequency $f$ of each row, a ranking order $r o_{c}$ of the criteria can be identified with $r o_{c}$ $=1$ for the criterion with the highest frequency. However, neither the frequency nor the ranking order is adequate for the calculation of weighting factors. While using the frequency could result in inconsistency and fuzziness of the data due to errors in the comparison, a calculation based on the ranking order would lead to an unrealistic weighting.

Thus, based on the SIMOS method, a modified approach has been developed. Depending on the difference of the frequency, the method adds additional fictitious ranks adjusting the number of rank for each criterion. Each fictional rank is correlated to the class. The size of the class $\mathrm{cl}$ is calculated according to (5) where the difference between the maximum $f_{\max }$ and minimum frequency $f_{\min }$ is divided by the existing number of ranks $m$. The identification of the class size allows determining the new rank order $r o_{c}^{\prime}$ by adding up the fictional ranks. Finally, with the definition of the new rank orders, the weighting factors can be determined. For this, the established rank exponent method will be applied (Stillwell et al., 1981).

$$
c l=\frac{f_{\max }-f_{\min }}{m} \wedge \quad r^{\prime}{ }_{c}=\left\{\begin{array}{c}
1:\left(f_{\text {ro }_{c-1}}-f_{\text {ro }_{c}}\right)<0 \\
r o^{\prime}{ }_{c-1}+\left\lceil\frac{f_{r o_{c-1}}-f_{r_{c}}}{c l}\right\rceil: \text { else }
\end{array}\right.
$$

The formula producing the normalized weighting factors $w_{c}$ is shown in (6) where $m^{\prime}$ stands for the new number of ranks and the $p$-parameter describes the weights for the most important criteria on a $0-1$ scale. While $p=0$ assigns equal weights to the criteria, an increasing $p$ leads to a steeper weight distribution. The parameter has to be determined by an iterative procedure. Weighting factors, which represent the preferences for each criterion, can be used in combination with the evaluation model to calculate the technological value. The following section presents an approach to define a key figure for the technological assessment of a joining technology. 


$$
w_{c}=\frac{\left(m^{\prime}-r o^{\prime}{ }_{c}+1\right)^{p}}{\sum_{c=1}^{n}\left(m^{\prime}-r o^{\prime}{ }_{c}+1\right)^{p}}
$$

\section{Static and dynamic assessment}

The evaluation and weighting model necessitates a key figure, which consolidates the derived insights. Since the early stage of product development is characterized by insufficient product and process information (Figure 4), the assessment is subdivided into the static and dynamic assessment within the product development which allows a more accurate assessment with an increasing data availability.

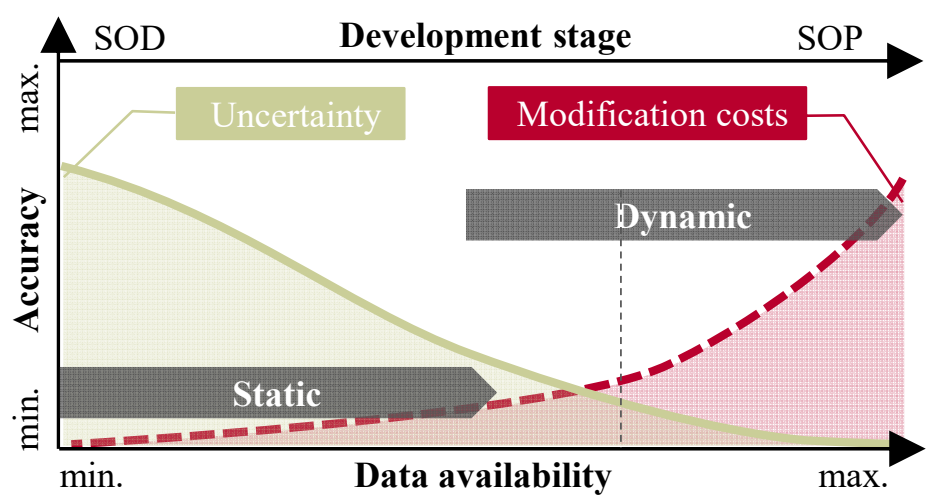

Figure 4. Data availability from start of development to start of production

\subsection{Static assessment figure}

The static assessment figure $S F_{T}$ is a ratio figure demonstrating the technological value of the joining technology $(T)$. The calculation of $S F_{T}$ requires the technological value of the joining technology $V_{T}$. As described in (7), $V_{T}$ summarizes the values of the evaluation $x_{c}$ and weighting factors $w_{c}$ for all criteria identified from the evaluation and weighting model. However, the calculation of a ratio also necessitates a reference value. For this purpose, the reference point method can be applied to determine the theoretical optimum value $\left(V_{\max }\right)$ for a joining technology with the ideal evaluation $x_{\max }$.

$$
V_{T}=\sum_{c=1}^{n} x_{c, T} \cdot w_{c} \quad \forall T=\{1, \ldots, n\} \quad \wedge \quad V_{\max }=\sum_{c=1}^{n} x_{\max } \cdot w_{c}
$$

With identifying the technological value for the joining technology as well as the optimum technological value, finally, the static assessment figure can be determined with (8) where $S F_{T}$ can have a solution set between zero and one, while a value of one represents the optimum.

$$
S F_{T}=\left(\frac{V_{T}}{V_{\max }}\right) \quad \forall T=\{1, \ldots, n\}
$$

A benefit of the static approach is the possibility for a rapid assessment of a joining technology without necessitating any product information, which is mainly in the early phase of development only sporadic available. However, a weakness is the inaccuracy of the assessment due to not considering productspecific requirements.

\subsection{Dynamic assessment figure}

While the static assessment is beneficial for the early phase of development, the dynamic assessment can be applied in a development stage where already information about the product and its requirements exist for a more accurate assessment. Since the dynamic assessment figure $D F_{T}$ is component specific, the criteria require a differentiation between global $G$ and local criteria $L$, as well as, binding constraints $E$, as described in the evaluation model. Global criteria are exclusively static; however, local criteria and binding constraints as a subset of local criteria are component specific, thus dynamic.

The structure of the dynamic assessment can be described with three functions: the reward, the penalty, and the exclusion function. For example, the reward function $R\left(r_{c}, x_{c}\right)$ compares the characteristic $x_{c}$ with 
the requirement $r_{c}$ of the set of local criteria. The requirements can receive, similar to the characteristics, a value between zero and nine. In case, the characteristic meets the requirement for a criterion the technology will be rewarded through adjusting the $x_{c}$ to $x_{\max }$ according to (9). Hence, the technology will be seen as an ideal technology for that particular criterion.

The penalty function $P\left(r_{c}, x_{c}\right)$ also compares the characteristic $x_{c}$ with the requirement $r_{c}$ of the set of local criteria. However, the penalty function stated in (10) allows penalizing technologies, where the characteristics do not meet the requirements for a criterion. The penalty for a criterion depends on the difference between the characteristic and requirement to consider the additional effort for the required adjustments (e.g., constructive modification of flange width).

The third function is the exclusion function $\theta\left(r_{c}, x_{c}\right)$, which is described in (11). The function analyzes the set of binding constraints $E$ to allow an efficient selection of joining technologies, which ensure the technological feasibility. Thus, in case any characteristic $x_{c}$ does not meet the requirement $r_{c}$ of the set of binding constraints, the exclusion function receives a value of zero. Another possibility to receive the value zero could be if the penalty function would become equal or greater than the sum of the technological value of global and local criteria to eliminate joining technologies with a negative technological value.

$$
\begin{aligned}
& R\left(r_{c}, x_{c, T}\right)= \begin{cases}x_{\max }: r_{c} \leq x_{c, T} \\
x_{c, T}: \text { else }\end{cases} \\
& P\left(r_{c}, x_{c, T}\right)=\max \left(0, r_{c}-x_{c, T}\right) \\
& \theta\left(r_{c}, x_{c, T}\right)= \begin{cases}0, & \sum_{c \in E} \max \left(0, r_{c}-x_{c, T}\right) \neq 0 \quad \mathrm{~V} \\
1, & \sum_{c \in G}\left(x_{c, T} \cdot w_{c}\right)+\sum_{c \in L} w_{c}\left(R\left(r_{c}, x_{c}\right)-P\left(r_{c}, x_{c}\right)\right) \leq 0 \\
1, & \text { else }\end{cases}
\end{aligned}
$$

With the reward, penalty and exclusion function, the static assessment figure can be extended to determine the dynamic technological value according to (12). Similar to $S F_{T}$, the solution set of $D F_{T}$ can be between zero and one, while a value of one represents the optimum.

$$
D F_{T}=\left(\frac{\sum_{c \in G}\left(x_{c, T} \cdot w_{c}\right)+\sum_{c \in L} w_{c}\left(R\left(r_{c}, x_{c}\right)-P\left(r_{c}, x_{c}\right)\right)}{V_{\max }}\right) \cdot \theta\left(r_{c}, x_{c, T}\right) \quad \forall T=\{1, \ldots, n\}
$$

The effects of the static and dynamic assessment with varying characteristics are described for a local criterion (a) and a varying binding constraint (b) (Figure 5). The requirement of the joining problem for this particular criterion is $r_{c}=5$. Both graphs show the linear behavior of the static assessment, where the requirement of the component has no impact on the technological value. The dynamic assessment, on the other hand, describes in the first graph the reward function with an improvement and the penalty function with a degradation of the technological value. The optimum dynamic assessment for that criterion is realized once $r_{c} \leq x_{c}$, i.e., as soon as the requirements of a component are satisfied, the characteristics have no further impact on the technological value. The second graph (b) shows the effect of the exclusion function. As long as the binding constraint $r_{c} \in E$ cannot be satisfied, the technological value equals 0 . Similar to the first graph, the optimum is realized with $r_{c} \in L \leq x_{c}$.

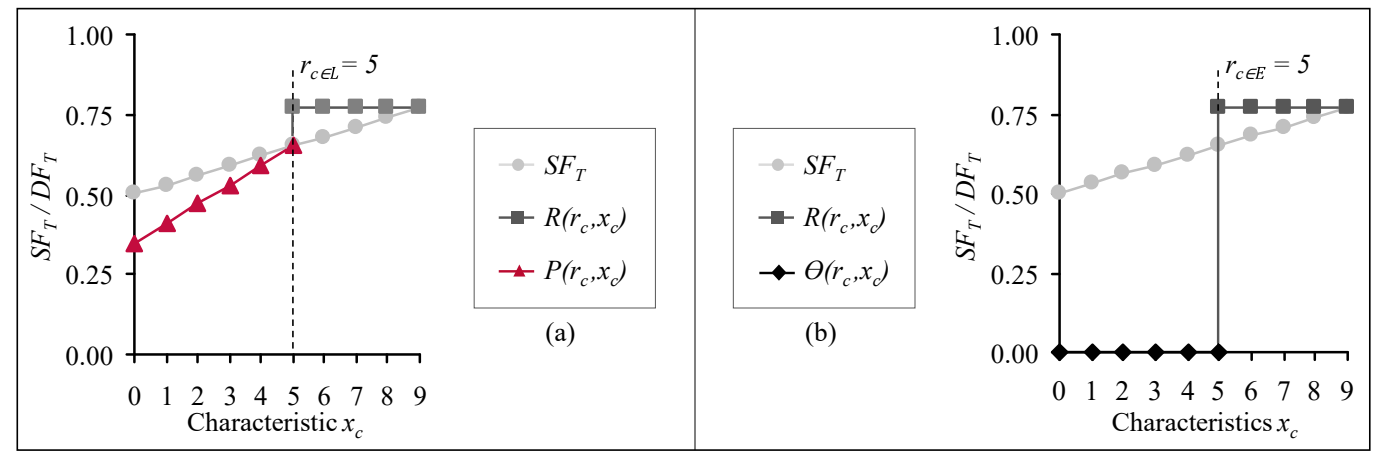

Figure 5. Effects of the reward and penalty function (a) and the exclusion function (b) 


\section{Application of the approach}

The research is applied to a fictitious use study in car body development for joining the panoramic roof (a) with the roof cross member (b) to demonstrate the potential of the presented approach for the technological assessment of joining technologies (Figure 6).

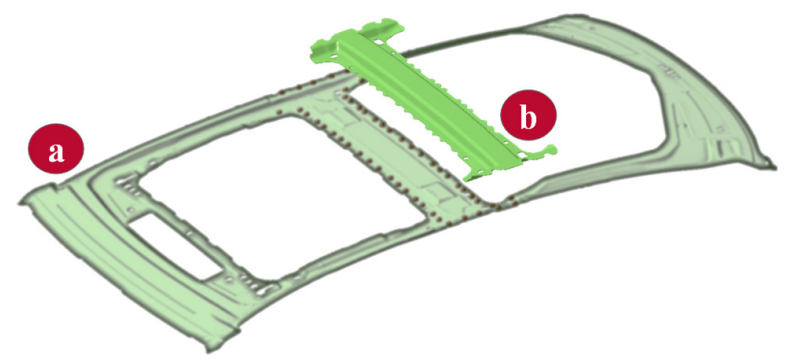

Figure 6. Joining of the panoramic roof (a) with a roof cross member (b)

For the technological assessment of the joining problem and identification of the optimum technology, the first step is the definition of criteria, which may have an impact on joining technologies. For the case study, an excerpt of possible criteria is listed and characterized in Table 5. The second step, which is the assignment of scale classes to the identified criteria, is also supplemented in the table.

Table 5. Characterization and scaling of criteria

\begin{tabular}{|c|c|c|c|c|}
\hline Criteria & Description & Nature & Possible classes & Scale (0-9) \\
\hline$C_{A}$ & Flange width & Quantitative & Local, Binding & Decreasing function (a) \\
\hline$C_{B}$ & Weight & Quantitative & Global & Decreasing function (a) \\
\hline$C_{C}$ & View-shed & Qualitative & Local, Binding & Step-function (c) \\
\hline$C_{D}$ & Friendliness & Qualitative & Global & linear-function (d) \\
\hline$C_{\mathrm{E}}$ & Size accuracy & Qualitative & Global & linear-function (d) \\
\hline$C_{F}$ & Accessibility & Qualitative & Local, Binding & Step-function (c) \\
\hline
\end{tabular}

The following step is the evaluation of the alternative technologies. For the transformation of the evaluation, the calculation of the scope for each criterion is required. Equation (13) shows exemplarily the calculation of the scope for criterion $C_{A}$ with a scale from zero to nine.

Table 6. Evaluation and transformation of criteria

\begin{tabular}{|c|c|c|c|c|c|c|c|c|c|c|c|c|}
\hline \multirow{2}{*}{$\begin{array}{l}\text { Criteria } \\
\text { Alternative }\end{array}$} & \multicolumn{2}{|c|}{$C_{A}$} & \multicolumn{2}{|c|}{$C_{B}$} & \multicolumn{2}{|c|}{$C_{C}$} & \multicolumn{2}{|c|}{$C_{D}$} & \multicolumn{2}{|c|}{$C_{E}$} & \multicolumn{2}{|c|}{$C_{F}$} \\
\hline & $v$ & $x$ & $v$ & $x$ & $v$ & $x$ & $v$ & $x$ & $v$ & $x$ & $v$ & $x$ \\
\hline Technology 1 & 18 & 6 & 45 & 0 & No & 0 & 5 & 5 & 5 & 5 & Yes & 9 \\
\hline Technology 2 & 17 & 7.5 & 15 & 6 & Yes & 9 & 6 & 6 & 9 & 9 & No & 0 \\
\hline Technology 3 & 16 & 9 & 5 & 8 & Yes & 9 & 8 & 8 & 7 & 7 & No & 0 \\
\hline Technology 4 & 18 & 6 & 0 & 9 & No & 0 & 9 & 9 & 7 & 7 & No & 0 \\
\hline Technology 5 & 22 & 0 & 0 & 9 & Yes & 9 & 8 & 8 & 4 & 4 & No & 0 \\
\hline
\end{tabular}

Finally, with the identification of the scope, the evaluated value of each technology can be transformed to normalized scaling points. For instance, $x_{c_{A, l}}$ shows the transformation of the value of criterion $C_{A}$ for joining technology 1 .

$$
s_{c_{A}}=\frac{22-16}{0-9}=-0.6 \overline{6} \quad \wedge \quad x_{c_{A, 1}}=\frac{18-22}{-0.6 \overline{6}}=6
$$

While the scope only varies between criteria, the transformation has to be performed for each criterion and each technology individually. Table 6 summarizes the results produced by the evaluation model. For the consideration of the preferences for each criterion, weighting factors have to be identified. 
Therefore, the pairwise comparison as an essential element of the weighting model compares all criteria among each other (Table 7).

Table 7. Excerpt of pairwise comparison for case study

\begin{tabular}{|c|c|c|c|c|c|c|}
\hline $\boldsymbol{i}$ & $\boldsymbol{C}_{\boldsymbol{A}}$ & $\boldsymbol{C}_{\boldsymbol{B}}$ & $\boldsymbol{C}_{\boldsymbol{C}}$ & $\boldsymbol{C}_{\boldsymbol{D}}$ & $\boldsymbol{C}_{\boldsymbol{E}}$ & $\boldsymbol{C}_{\boldsymbol{F}}$ \\
\hline$C_{A}$ & - & 1 & 1 & 1 & 0 & 0.5 \\
\hline$C_{B}$ & 0 & - & 0 & 0.5 & 0 & 0 \\
\hline$C_{C}$ & 0 & 1 & - & 1 & 0 & 0.5 \\
\hline$C_{D}$ & 0 & 0.5 & 0 & - & 0 & 0 \\
\hline$C_{E}$ & 1 & 1 & 1 & 1 & - & 1 \\
\hline$C_{F}$ & 0.5 & 1 & 0.5 & 1 & 0 & - \\
\hline
\end{tabular}

With the frequency of each criterion and its rank order, the size of the classes can be calculated according to (5). The class size for the case study is calculated in (14) with the data provided from Table 8. As described in (5), to determine the new order of ranks necessitates adding fictional ranks. For instance, the new rank $\left(r o^{\prime}\right)$ for the second-ranked criterion $C_{A}$ is calculated by adding one fictional rank.

$$
c l=\frac{5-0.5}{5}=0.9 \quad \wedge \quad r^{\prime}{ }_{c_{A}}=1+\left\lceil\frac{5-3-5}{0.9}\right\rceil=3
$$

Table 8 gives an overview of the new ranks for the individual criteria. With the additional fictional ranks, the total number of ranks has been changed from 5 to 8 . The findings can be used to produce normalized weight factors with the rank exponent method according to (6). Exemplary, the weighting factor for criteria $C_{A}$ is illustrated in (15). For the use study, the parameter $p$ has been set to one, which results in a steep weight distribution.

$$
w_{c_{A}}=\frac{(8-3+1)^{1}}{6^{1}+1^{1}+4^{1}+1^{1}+8+5^{1}}=0.24
$$

Table 8 also shows the resulting weighting factors $\left(w_{c}\right)$ for each criterion. The criteria weight for a joining element $\left(C_{B}\right)$ and service friendliness $\left(C_{D}\right)$ indicate only limited importance. However, the criteria size accuracy $\left(C_{E}\right)$ and flange width $\left(C_{A}\right)$ have, besides the criteria view-shed $\left(C_{C}\right)$ and accessibility $\left(C_{F}\right)$, a relatively high impact on the decision-making of joining technologies.

Table 8. Analysis of pairwise comparison

\begin{tabular}{|c|c|c|c|c|c|}
\hline $\boldsymbol{r} \boldsymbol{o}_{\boldsymbol{c}}$ & Criteria & $\boldsymbol{f}_{\boldsymbol{c}}$ & $\boldsymbol{f}_{\boldsymbol{r} \boldsymbol{c}-\boldsymbol{1}}-\boldsymbol{f}_{\boldsymbol{r o}_{\boldsymbol{c}}}$ & $\boldsymbol{r o}_{\boldsymbol{c}}{ }_{\boldsymbol{c}}$ & $\boldsymbol{w}_{\boldsymbol{c}_{\boldsymbol{A}}}$ \\
\hline 1 & $C_{E}$ & 5 & -5 & 1 & $32 \%$ \\
\hline 2 & $C_{A}$ & 3.5 & 1.5 & 3 & $24 \%$ \\
\hline 3 & $C_{F}$ & 3 & 0.5 & 4 & $20 \%$ \\
\hline 4 & $C_{C}$ & 2.5 & 0.5 & 5 & $16 \%$ \\
\hline 5 & $C_{B}$ & 0.5 & 2 & 8 & $4 \%$ \\
\hline 5 & $C_{D}$ & 0.5 & 0 & 8 & $4 \%$ \\
\hline
\end{tabular}

The completion of the evaluation of the joining technologies and the weighting of the criteria allows determining the static technological value presented in (8). The calculation of the static assessment figure is performed exemplarily in (16) for technology 5.

$$
S F_{5}=\left(\frac{3.4}{9}\right)=0.38
$$

For the dynamic assessment, the requirements of the joining problem have to be considered. An excerpt of possible requirements shows Table 9. While flange width represents a binding constraint, view-shed and one-sided accessibility are both classified as local requirements. The definition of requirements allows a dynamic assessment of the technologies for the specific joining problem. 
Table 9. Identification and definition of requirements

\begin{tabular}{|c|c|c|c|}
\hline Criteria & Description & $\boldsymbol{r}_{\boldsymbol{c}}$ & Class of $\boldsymbol{r}_{\boldsymbol{c}}$ \\
\hline $\mathrm{C}_{\mathrm{A}}$ & Flange width of $18 \mathrm{~mm}$ required & 6 & Binding \\
\hline $\mathrm{C}_{\mathrm{C}}$ & View-shed not required & 0 & Local \\
\hline $\mathrm{C}_{\mathrm{F}}$ & One-sided accessibility preferred & 9 & Local \\
\hline
\end{tabular}

Analog to the static assessment, the dynamic assessment figure is performed exemplarily for technology 5 in (17) indicating a technological value of zero. The trigger of the exclusion function is caused by the binding constraint of $C_{A}$, which technology 5 cannot satisfy.

$$
D F_{5}=\left(\frac{1.96+1.44-1.80}{9}\right) \cdot 0=0
$$

The findings of the static and dynamic assessment are shown in Figure 7. While an assessment figure of zero represents a technology with no technological feasibility, an increasing figure leads to a growing technological value. For example, in the presented cobweb chart, technology 2 appears to be the relatively best technology within the static assessment. However, the dynamic assessment points out that technology 1 is the technology with the highest technological value for the specific joining problem. Hence, under consideration of the technological criteria technology 1 should be chosen for joining the panoramic roof with the roof cross member.

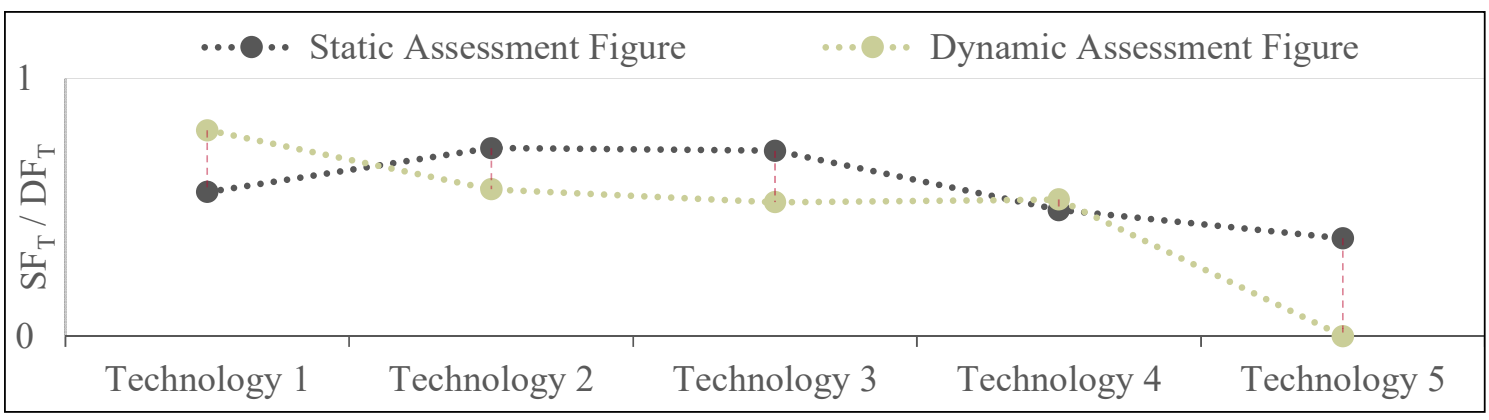

Figure 7. Results of the technological assessment

\section{Conclusion and outlook}

The presented approach allows a transparent and optimized decision-making for joining technologies due to the consideration of technological criteria. For the technological assessment, an evaluation model has been developed and linked with a weighting model to represent realistic preferences of each criterion in the automobile body development. Both models can satisfy all requirements conducted from the stakeholder-analysis for the evaluation and weighting of non-monetary criteria.

For the concluding assessment, the derived insights have been concentrated to a static and a dynamic key figure. While the static approach allows an assessment in an early development stage, the dynamic approach can be applied in a later development stage where already information about the joining problem exist to enable a more accurate assessment. Finally, for a validation of the presented approach, the theoretical methodology has been applied to a case study in car body development.

Nevertheless, efficient planning and manufacturing processes necessitate a multi-criteria decision analysis (MCDA) which considers besides technological also economic and ecological criteria of joining technologies. Thus, an economic and ecological model has to be developed which analyzes lifecycle costs and the environmental impact of joining technologies to determine the actual optimum joining technology for specific joining problems. The multi-criteria assessment allows a holistic and transparent overview of the impact for the individual solutions to support the decision-making of an engineer. To further increase the acceptance for the assessment approach of an engineer, the individual key figures for the economic, ecological, and technological dimension can be consolidated to one performance indicator as a "price label" indicating the total value of a joining technology. 


\section{References}

Breiing, A. and Knosala, R. (1997), “Teoretische Grundlagen”, In: Breiing, A. and Knosala, R. (Ed.), Bewerten technischer Systeme, Springer Berlin, Berlin, pp. 49-208. https://doi.org/10.1007/978-3-642-59229-4

Choudry, S., Müller, S., Alber, U., Riedel, F. and Landgrebe, D. (2018), “A Multidimensional Assessment and Selection Methodology: Optimized Decision-making of Joining Technologies in Automobile Body Development", 15th Global Conference on Sustainable Manufacturing, Haifa, Israel, September 25-27, 2018, Procedia Manufacturing. https://doi.org/10.1016/j.promfg.2018.02.122

Fink, K., Ploder, C. and Roithmayr, F. (2006), Wirtschaftsinformatik als Schlüssel zum Unternehmenserfolg, Deutscher Universitäts-Verlag, Wiesbaden, p. 105. https://doi.org/10.1007/978-3-8350-9122-1

Fodor, J. and Roubens, M. (2011), "Valued binary relations", In: Fuzzy Preference Modelling and Multicriteria Decision Support, Theory and Decision Library (Series D: System Theory, Knowledge Engineering and Problem Solving), Vol. 14, Springer, Dordrecht, pp. 56-57. https://doi.org/10.1007/978-94-017-1648-2

Hahn, O., Janzen, V., Meschut, G., Olfermann, T. and Süllentrop, S. (2013), "Fügeverfahren in hybriden Leichtbausystemen", In: Friedrich, H. (Ed.), Leichtbau in der Fahrzeugtechnik, Springer Vieweg, Wiesbaden, pp. 623-624. https://doi.org/10.1007/978-3-8348-2110-2

Kühnapfel, J. (2014), Nutzwertanalysen in Marketing und Vertrieb, Springer-Gabler, Wiesbaden, pp. 5-7. https://doi.org/10.1007/978-3-658-05509-7

Miller, G. (1994), "The magical number seven, plus or minus two: Some limits on our capacity for processing information", Psychological Review, Vol. 101 No. 2, pp. 343-352. https://doi.org/10.1037//0033295x.101.2.343

Nedjah, N. and Macedo Mourelle, L. (2005), “A Qualitative Approach for Symbolic Data Manipulation Under Uncertainty”, In: Nedjah N. and Macedo Mourelle L. (Ed.), Fuzzy systems engineering, Studies in Fuzziness and Soft Computing, Vol. 181, Springer, Berlin, p. 35. https://doi.org/10.1007/b102051

Porst, R. (2014), Fragebogen: Ein Arbeitsbuch, Lehrbuch, Springer VS, Wiesbaden. https://doi.org/10.1007/9783-658-02118-4

Saaty, T. (2008), "Relative measurement and its generalization in decision making why pairwise comparisons are central in mathematics for the measurement of intangible factors the analytic hierarchy/network process", Revista de la Real Academia de Ciencias Exactas, Fisicas y Naturales. Serie A. Matematicas, Vol. 102 No. 2, pp. 251-318. https://doi.org/10.1007/bf03191825

Siskos, E. and Tsotsolas, N. (2015), "Elicitation of criteria importance weights through the Simos method: A robustness concern", European Journal of Operational Research, Vol. 246 No. 2, pp. 543-553. https://doi.org/10.1016/j.ejor.2015.04.037

Stillwell, W., Seaver, D. and Edwards, W. (1981), "A comparison of weight approximation techniques in multiattribute utility decision making”, Organizational Behavior and Human Performance, Vol. 28 No. 1, pp. 62-77. https://doi.org/10.1016/0030-5073(81)90015-5

Teske, L., Großmann, H. and Timm, H. (2013), "Materialauswahl und Leichtbau”, In: Braess, H. and Seifert, U. (Eds.), Vieweg Handbuch Kraftfahrzeugtechnik, Springer Vieweg, Wiesbaden, pp. 386-388. https://doi.org/10.1007/978-3-658-01691-3

Tullis, T. and Albert, W. (2008), “Self-Reported Metrics”, In: Tullis, T. and Albert, W. (Eds.), Measuring the user experience: Collecting, Analyzing, and Presenting Usability Metrics, Morgan Kaufmann, Amsterdam, pp. 125125. https://doi.org/10.1016/B978-0-12-373558-4.00006-6

VDI 2225 (1998), Blatt 3 - Design engineering methodics - Engineering design at optimum cost - Valuation of costs, Verein Deutscher Ingenieure.

Saphir Ahmad Choudry, M.Sc.

Chemnitz University of Technology, Institute for Machine Tools and Production Processes

Reichenhainer Str. 70, 09126 Chemnitz, Germany

Email: saphir-a.choudry@mb.tu-chemnitz.de 